\title{
EXPLICIT STABILITY TESTS FOR LINEAR NEUTRAL DELAY EQUATIONS USING INFINITE SERIES
}

\author{
LEONID BEREZANSKY AND ELENA BRAVERMAN
}

\begin{abstract}
We obtain new explicit exponential stability conditions for the linear scalar neutral equation with two bounded delays $(x(t)-a(t) x(g(t)))^{\prime}+b(t) x(h(t))=0$, where $|a(t)| \leq A_{0}<1$, $0<b_{0} \leq b(t) \leq B_{0}$, assuming that all parameters of the equation are measurable functions.

To analyze exponential stability, we apply the Bohl-Perron theorem and a reduction of a neutral equation to an equation with an infinite number of non-neutral delay terms. This method has never been used before for this neutral equation; its application allowed to omit a usual restriction $|a(t)|<\frac{1}{2}$ in known asymptotic stability tests and consider variable delays.
\end{abstract}

1. Introduction and Preliminaries. Neutral differential equations have many applications in control theory, ecology, biology, physics, see, for example, [4, $\mathbf{6}, \mathbf{8}, \mathbf{9}, \mathbf{1 0}]$. The aim of the present paper is to obtain new explicit exponential stability conditions for the equation

$$
(x(t)-a(t) x(g(t)))^{\prime}=-b(t) x(h(t)) .
$$

Its particular case with constant delays

$$
(x(t)-a(t) x(t-\sigma))^{\prime}+b(t) x(t-\tau)=0,
$$

where $\tau, \sigma>0, a, b \in C\left(\left[t_{0}, \infty\right), \mathbb{R}\right), b(t) \geq 0$, has been extensively investigated [11, 12].

Proposition 1. [12] If $\int_{t_{0}}^{\infty} b(s) d s=+\infty,|a(t)| \leq A_{0}<1$ and $\limsup _{t \rightarrow \infty} \int_{t-\tau}^{t} b(s) d s<\frac{3}{2}-2 A_{0}\left(2-A_{0}\right)$ then equation (1.2) is asymptotically stable.

In the non-neutral case $a(t) \equiv 0$, Proposition 1 turns into the sharp stability result with the constant $\frac{3}{2}$. There are several improvements and extensions of Proposition 1, we cite the test obtained in [11.

Proposition 2. [11] Let $b(t) \geq 0, \int_{t_{0}}^{\infty} b(s) d s=+\infty,|a(t)| \leq A_{0}<1$, and at least one of the following conditions hold:

$$
\begin{aligned}
& \text { a) } A_{0}<\frac{1}{4}, \limsup _{t \rightarrow \infty} \int_{t-\tau}^{t} b(s) d s<\frac{3}{2}-2 A_{0} \\
& \text { b) } \frac{1}{4} \leq A_{0}<\frac{1}{2}, \limsup _{t \rightarrow \infty} \int_{t-\tau}^{t} b(s) d s<\sqrt{2\left(1-2 A_{0}\right)} .
\end{aligned}
$$

Then equation (1.2) is asymptotically stable.

It is easy to see that both propositions assume $|a(t)|<\frac{1}{2}$. Additional restrictions are that the delays are constant, coefficients $a(t)$ and $b(t)$ are continuous functions. In the present paper, we omit some of these restrictions.

1991 AMS Mathematics subject classification. 34K40, 34K20, 34K06.

Keywords and phrases. Neutral equations in the Hale form, uniform exponential stability, Bohl-Perron theorem, variable delays, explicit stability conditions.

Partially supported by the NSERC research grant RGPIN-2015-05976.

Received by the editors July 5, 2018; revised September 6, 2018. 
To analyze exponential stability, we apply the Bohl-Perron theorem and a reduction of a neutral equation to an equation with an infinite number of non-neutral delay terms. This method has never been used before for neutral equation (1.1).

We consider (1.1) under a number of the following assumptions:

(a1) $a, b, g, h$ are Lebesgue measurable on $[0,+\infty)$, and there exist positive constants $A_{0}, b_{0}, B_{0}$ such that $|a(t)| \leq A_{0}<1,0<b_{0} \leq b(t) \leq B_{0}$

(a2) mes $\Omega=0 \Longrightarrow$ mes $g^{-1}(\Omega)=0$, where mes $\Omega$ is the Lebesgue measure of the set $\Omega$;

(a3) $g(t) \leq t, h(t) \leq t, \lim \sup _{t \rightarrow \infty} g(t)=\infty, \limsup _{t \rightarrow \infty} h(t)=\infty$;

(a4) $0 \leq t-g(t) \leq \sigma, 0 \leq \delta \leq t-h(t) \leq \tau, t \geq t_{0}$ for some $t_{0} \geq 0$ and $t \geq t_{0}$.

Together with (1.1) we consider for each $t_{0} \geq 0$ an initial value problem

$$
(x(t)-a(t) x(g(t)))^{\prime}+b(t) x(h(t))=f(t), t \geq t_{0}, x(t)=\varphi(t), t \leq t_{0}
$$

and assume that for $f$ and $\varphi$ the following condition holds:

(a5) $f:\left[t_{0},+\infty\right) \rightarrow \mathbb{R}$ is Lebesgue measurable locally essentially bounded, $\varphi:\left(-\infty, t_{0}\right) \rightarrow \mathbb{R}$ is a Borel measurable bounded function.

Definition 1. A function $x: \mathbb{R} \rightarrow \mathbb{R}$ is called a solution of problem (1.3) if the difference $x(t)-a(t) x(g(t))$ is absolutely continuous on each interval $\left[t_{0}, c\right], x$ satisfies the equation in (1.3) for almost all $t \in\left[t_{0},+\infty\right)$ and the initial condition in (1.3) for $t \leq t_{0}$.

There exists a unique solution of problem (1.3) if conditions (a1)-(a3),(a5) hold, see [5, $\mathbf{8}$.

Consider the initial value problem for the equation with one non-neutral delay term

$$
x^{\prime}(t)+b(t) x(h(t))=f(t), t \geq t_{0}, x(t)=0, t \leq t_{0},
$$

where $b(t), f(t)$ and $h(t) \leq t$ are Lebesgue measurable locally essentially bounded functions.

Definition 2. For each $s \geq t_{0}$ the solution $X(t, s)$ of the problem

$$
x^{\prime}(t)+b(t) x(h(t))=0, t \geq t_{0}, x(t)=0, t<s, x(s)=1
$$

is called a fundamental function of equation (1.4). We assume $X(t, s)=0$ for $0 \leq t<s$.

Lemma 1. 2] Let (a1)-(a3),(a5) hold. The solution of problem 1.4) can be presented in the form

$$
x(t)=\int_{t_{0}}^{t} X(t, s) f(s) d s
$$

Definition 3. Equation (1.1) is (uniformly) exponentially stable if there exist positive numbers $M$ and $\gamma$ such that the solution of problem (1.3) with $f \equiv 0$ has the estimate

$$
|x(t)| \leq M e^{-\gamma\left(t-t_{0}\right)} \sup _{t \in\left(-\infty, t_{0}\right]}|\varphi(t)|, t \geq t_{0},
$$

where $M$ and $\gamma$ do not depend on $t_{0} \geq 0$ and $\varphi$.

Next, we present the Bohl-Perron theorem.

Lemma 2. [5, Theorem 6.1] Let (a1)-(a2),(a4) and (a5) hold. Assume that the solution of the problem

$$
(x(t)-a(t) x(g(t)))^{\prime}+b(t) x(h(t))=f(t), x(t)=0, t \leq t_{0}
$$

is essentially bounded on $\left[t_{0},+\infty\right)$ for any essentially bounded $f:\left[t_{0},+\infty\right) \rightarrow \mathbb{R}$. Then equation (1.1) is uniformly exponentially stable. 
Remark 1. In Lemma 2 we can consider boundedness of solutions not for all essentially bounded functions $f:\left[t_{0},+\infty\right) \rightarrow \mathbb{R}$ but only for those which satisfy $f(t)=0, t \in\left[t_{0}, t_{1}\right)$, for any fixed $t_{1}>t_{0}$, see [3]. We will further apply this fact in the paper without an additional reference.

Consider now a linear equation with a measurable single delay and a locally essentially bounded nonnegative coefficient

$$
x^{\prime}(t)+b(t) x\left(h_{0}(t)\right)=0, b(t) \geq 0,0 \leq t-h_{0}(t) \leq \tau_{0} .
$$

Let $X_{0}(t, s)$ be a fundamental function of (1.6).

Lemma 3. [3] Assume that $X_{0}(t, s)>0, t \geq s \geq t_{0}$. Then

$$
\int_{t_{0}+\tau_{0}}^{t} X_{0}(t, s) b(s) d s \leq 1
$$

Lemma 4. [3, 7] If for some $t_{0} \geq 0$ the inequality $\int_{h_{0}(t)}^{t} b(s) d s \leq \frac{1}{e}$ holds for $t \geq t_{0}$ then $X_{0}(t, s)>0$ for $t \geq s \geq t_{0}$. If in addition $b(t) \geq b_{0}>0$ then equation (1.6) is exponentially stable.

For a fixed bounded interval $I=\left[t_{0}, t_{1}\right]$, consider the space $L_{I}:=L_{\infty}\left[t_{0}, t_{1}\right]$ of all essentially bounded on $I$ functions with the norm $|y|_{I}=\operatorname{ess}_{\sup _{t \in I}}|y(t)|$, for an unbounded interval denote $\|f\|_{\left[t_{0},+\infty\right)}=\underset{t \geq t_{0}}{\operatorname{ess} \sup }|f(t)|$, $E$ is the identity operator. Define a linear operator on the space $L_{I}$ as

$$
(S y)(t)= \begin{cases}a(t) y(g(t)), & g(t) \geq t_{0}, \\ 0, & g(t)<t_{0},\end{cases}
$$

where $|a(t)| \leq A_{0}<1$ is Lebesgue measurable, $g(t) \leq t$ is measurable satisfying (a2).

Lemma 5. [1] $E-S$ is invertible in the space $L_{I}$ for any $t_{1}>t_{0},(E-S)^{-1}=\sum_{j=0}^{\infty} S^{j}$, where $S^{0}=E$, and the operator norm satisfies

$$
\left\|(E-S)^{-1}\right\|_{L_{I} \rightarrow L_{I}} \leq \frac{1}{1-\|a\|_{I}}
$$

2. Stability Results. Consider initial value problem (1.5) with $\|f\|_{\left[t_{0},+\infty\right)}<+\infty$. Further, we assume that a product equals one if it contains no factors, for example, $\prod_{k=0}^{-1}=1$.

Define

$$
\tau_{0}=\frac{1-\|a\|_{\left[t_{0},+\infty\right)}}{e\|b\|_{\left[t_{0},+\infty\right)}}
$$

Theorem 1. Assume that (a1),(a2),(a4) hold and there is $\alpha \in[0,1]$ such that for $t \geq t_{0}, a(t) \geq a_{0}>0$, $\alpha \tau_{0} \leq \delta$ and

$$
\tau\|b\|_{\left[t_{0}, \infty\right)}+\frac{\sigma\|a\|_{\left[t_{0}, \infty\right)}\|b\|_{\left[t_{0}, \infty\right)}\left(1-a_{0}\right)}{\left(1-\|a\|_{\left[t_{0}, \infty\right)}\right)^{2}}<\left(1-\|a\|_{\left[t_{0}, \infty\right)}\right)\left(1+\frac{\alpha}{e}\right) .
$$

Then equation (1.1) is uniformly exponentially stable. 
Proof. Consider initial value problem (1.5) with $\|f\|_{\left[t_{0},+\infty\right)}<+\infty, f(t)=0$ for $t \in\left[t_{0}, t_{0}+\tau\right]$ and let $I=\left[t_{0}, t_{1}\right]$ for some $t_{1}>t_{0}+\tau$. By (1.7) the solution $x$ of (1.5) and the derivative of the function $y(t)=x(t)-a(t) x(g(t))$ satisfy $y^{\prime}(t)=-b(t) x(h(t))+f(t), x=(E-S)^{-1} y$ and

$$
|x|_{I} \leq \frac{1}{1-\|a\|_{\left[t_{0},+\infty\right)}}|y|_{I},\left|y^{\prime}\right|_{I} \leq \frac{\|b\|_{\left[t_{0},+\infty\right)}}{1-\|a\|_{\left[t_{0},+\infty\right)}}|y|_{I}+\|f\|_{\left[t_{0},+\infty\right)} .
$$

By Lemma 5. (1.5) is equivalent to the problem for the equation with an infinite number of delays

$$
y^{\prime}(t)=-b(t) \sum_{j=0}^{\infty} \prod_{k=0}^{j-1} a\left(h\left(g^{[k]}(t)\right)\right) y\left(h\left(g^{[j]}(t)\right)\right)+f(t), y(t)=0, t \leq t_{0},
$$

where

$$
g^{[0]}(t)=t, g^{[1]}(t)=g(t), g^{[k]}(t)=g\left(g^{[k-1]}(t)\right), k \in \mathbb{N}
$$

Denote

$$
B(t)=b(t) \sum_{j=0}^{\infty} \prod_{k=0}^{j-1} a\left(h\left(g^{[k]}(t)\right)\right)
$$

where we can assume that $a(t)=a_{0}$ for $t<t_{0}$. Consider the delay equation

$$
x^{\prime}(t)+B(t) x\left(t-\alpha \tau_{0}\right)=0,
$$

where $B$ is defined in (2.6) and $\tau_{0}$ in (2.1).

Using the bounds for $a$ and $b$, we obtain $\frac{b_{0}}{1-a_{0}} \leq B(t) \leq \frac{\|b\|_{\left[t_{0}, \infty\right)}}{1-\|a\|_{\left[t_{0}, \infty\right)}}$.

Equation (2.4) can be rewritten in the form

$$
y^{\prime}(t)+B(t) y\left(t-\alpha \tau_{0}\right)=b(t) \sum_{j=0}^{\infty} \prod_{k=0}^{j-1} a\left(h\left(g^{[k]}(t)\right)\right) \int_{h\left(g^{[j]}(t)\right)}^{t-\alpha \tau_{0}} y^{\prime}(\xi) d \xi+f(t) .
$$

Since $B(t) \geq b_{0}$ and $\alpha \tau_{0} B(t) \leq \frac{1}{e}$, by Lemma 4 equation (2.7) is exponentially stable, and its fundamental function is positive: $X_{0}(t, s)>0, t \geq s \geq t_{0}$.

We have

$$
y(t)=\int_{t_{0}}^{t} X_{0}(t, s) B(s)\left(\frac{b(s)}{B(s)} \sum_{j=0}^{\infty} \prod_{k=0}^{j-1} a\left(h\left(g^{[k]}(s)\right)\right) \int_{h\left(g^{[j]}(s)\right)}^{s-\alpha \tau_{0}} y^{\prime}(\xi) d \xi\right) d s+f_{1}(t),
$$

where $\left\|f_{1}\right\|_{\left[t_{0}, \infty\right)} \leq \int_{t_{0}}^{t} X_{0}(t, s)|f(s)| d s<\infty$. Next, the arguments satisfy

$$
\begin{gathered}
t-g(t) \leq \sigma, t-g^{[2]}(t)=t-g(t)+(g(t)-g(g(t))) \leq 2 \sigma, \ldots, t-g^{[n]}(t) \leq n \sigma, \\
t-h(t) \leq \tau, t-h(g(t))=t-g(t)+(g(t)-h(g(t))) \leq \sigma+\tau, \ldots, t-h\left(g^{[n]}(t)\right) \leq n \sigma+\tau, \\
t-h(t) \geq \delta, t-h(g(t))=t-g(t)+(g(t)-h(g(t))) \geq \delta, \ldots, t-h\left(g^{[n]}(t)\right) \geq \delta,
\end{gathered}
$$

hence $t-h\left(g^{[n]}(t)\right) \geq \delta \geq \alpha \tau_{0}$. Therefore $t-\alpha \tau_{0}-h\left(g^{[n]}(t)\right)=t-h\left(g^{[n]}(t)\right)-\alpha \tau_{0} \geq \delta-\alpha \tau_{0} \geq 0$. 
Then for $t \in I$, by (2.6),

$$
\begin{aligned}
& \frac{b(s)}{B(s)} \sum_{j=0}^{\infty} \prod_{k=0}^{j-1} a\left(h\left(g^{[k]}\right)(s)\right) \int_{h\left(g^{[j]}(s)\right)}^{s-\alpha \tau_{0}}\left|y^{\prime}(\xi)\right| d \xi \\
\leq & \frac{b(s)}{B(s)} \sum_{j=0}^{\infty} \prod_{k=0}^{j-1} a\left(h\left(g^{[k]}\right)(s)\right)\left(\tau-\alpha \tau_{0}+j \sigma\right)\left|y^{\prime}\right|_{I} \\
= & {\left[\frac{\left(\tau-\alpha \tau_{0}\right) b(s)}{B(s)} \sum_{j=0}^{\infty} \prod_{k=0}^{j-1} a\left(h\left(g^{[k]}\right)(s)\right)+\frac{b(s)}{B(s)} \sum_{j=0}^{\infty} \prod_{k=0}^{j-1} a\left(h\left(g^{[k]}\right)(s)\right) j \sigma\right]\left|y^{\prime}\right|_{I} } \\
= & {\left[\frac{\left(\tau-\alpha \tau_{0}\right) B(s)}{B(s)}+\frac{b(s)}{B(s)} \sum_{j=0}^{\infty} \prod_{k=0}^{j-1} a\left(h\left(g^{[k]}\right)(s)\right) j \sigma\right]\left|y^{\prime}\right|_{I} } \\
\leq & {\left[\tau-\alpha \tau_{0}+\left(1-a_{0}\right)\|a\|_{\left[t_{0}, \infty\right)} \sigma \sum_{j=1}^{\infty} j\|a\|_{\left[t_{0}, \infty\right)}^{j-1}\right]\left|y^{\prime}\right|_{I} } \\
\leq & {\left[\tau-\alpha \tau_{0}+\frac{\sigma\|a\|_{\left[t_{0}, \infty\right)}\left(1-a_{0}\right)}{\left(1-\|a\|_{\left[t_{0}, \infty\right)}\right)^{2}}\right] \frac{\|b\|_{\left[t_{0}, \infty\right)}}{1-\|a\|_{\left[t_{0}, \infty\right)}}|y|_{I}+M_{1}, }
\end{aligned}
$$

where the finite constant $M_{1}$ does not depend on $I$, and the transition between the fourth and the fifths rows of the inequality is due to

$$
\frac{b(s)}{B(s)}=\left(\sum_{j=0}^{\infty} \prod_{k=0}^{j-1} a\left(h\left(g^{[k]}\right)(s)\right)\right)^{-1} \leq\left(\sum_{j=0}^{\infty} a_{0}^{j}\right)^{-1}=1-a_{0} .
$$

By Lemma 3 the solution of problem (1.5) satisfies

$$
|y|_{I} \leq\left[\tau-\alpha \tau_{0}+\frac{\sigma\|a\|_{\left[t_{0}, \infty\right)}\left(1-a_{0}\right)}{\left(1-\|a\|_{\left[t_{0}, \infty\right)}\right)^{2}}\right] \frac{\|b\|_{\left[t_{0}, \infty\right)}}{1-\|a\|_{\left[t_{0}, \infty\right)}}|y|_{I}+M_{2},
$$

where the constant $M_{2}$ does not dependent on $I$. Inequality (2.2) implies

$$
\left[\tau-\alpha \tau_{0}+\frac{\sigma\|a\|_{\left[t_{0}, \infty\right)}\left(1-a_{0}\right)}{\left(1-\|a\|_{\left[t_{0}, \infty\right)}\right)^{2}}\right] \frac{\|b\|_{\left[t_{0}, \infty\right)}}{1-\|a\|_{\left[t_{0}, \infty\right)}}<1 .
$$

Hence $|y(t)| \leq M$ for $t \geq t_{0}$, for some constant $M$ which does not depend on the interval $I$. Therefore $x$ is a bounded function on $\left[t_{0}, \infty\right)$. By Lemma 2, equation (1.1) is uniformly exponentially stable.

Assuming $\alpha=1$ and $\alpha=0$ in Theorem 1 , we get the following stability tests.

Corollary 1. Assume that (a1),(a2),(a4) are satisfied, for $t \geq t_{0}, a(t) \geq a_{0}>0$ and at least on of the following conditions holds:

$$
\begin{aligned}
& \text { a) } \tau_{0} \leq \delta, \tau\|b\|_{\left[t_{0}, \infty\right)}+\frac{\sigma\|a\|_{\left[t_{0}, \infty\right)}\|b\|_{\left[t_{0}, \infty\right)}\left(1-a_{0}\right)}{\left(1-\|a\|_{\left[t_{0}, \infty\right)}\right)^{2}}<\left(1-\|a\|_{\left[t_{0}, \infty\right)}\right)\left(1+\frac{1}{e}\right) \\
& \text { b) } \tau\|b\|_{\left[t_{0}, \infty\right)}+\frac{\sigma\|a\|_{\left[t_{0}, \infty\right)}\|b\|_{\left[t_{0}, \infty\right)}\left(1-a_{0}\right)}{\left(1-\|a\|_{\left[t_{0}, \infty\right)}\right)^{2}}<1-\|a\|_{\left[t_{0}, \infty\right)} .
\end{aligned}
$$

Then equation (1.1) is uniformly exponentially stable. 
Corollary 2. Assume that (a1)-(a3) hold, $t-g(t) \leq \sigma$, there exists $\lim _{t \rightarrow \infty}(t-h(t))=\tau$ and for some $t_{0} \geq 0$ and $\alpha \in[0,1]$, for $t \geq t_{0}$, we have $a(t) \geq a_{0}>0$ and

$$
\frac{\alpha\left(1-\|a\|_{\left[t_{0}, \infty\right)}\right)}{e}<\tau\|b\|_{\left[t_{0}, \infty\right)}<\left(1-\|a\|_{\left[t_{0}, \infty\right)}\right)\left(1+\frac{\alpha}{e}\right)-\frac{\sigma\|a\|_{\left[t_{0}, \infty\right)}\|b\|_{\left[t_{0}, \infty\right)}\left(1-a_{0}\right)}{\left(1-\|a\|_{\left[t_{0}, \infty\right)}\right)^{2}} .
$$

Then equation (1.1) is uniformly exponentially stable.

Proof. Since $\lim _{t \rightarrow \infty}(t-h(t))=\tau$, for any $\varepsilon>0$, in particular, for

$$
\varepsilon<\min \left\{\tau-\frac{\alpha\left(1-\|a\|_{\left[t_{0}, \infty\right)}\right)}{e\|b\|_{\left[t_{0}, \infty\right)}}, \frac{1-\|a\|_{\left[t_{0}, \infty\right)}}{\|b\|_{\left[t_{0}, \infty\right)}}\left(1+\frac{\alpha}{e}\right)-\frac{\sigma\|a\|_{\left[t_{0}, \infty\right)}\left(1-a_{0}\right)}{\left(1-\|a\|_{\left[t_{0}, \infty\right)}\right)^{2}}\right\}
$$

there exists $t_{1} \geq t_{0}$ such that $\tau-\varepsilon \leq t-h(t) \leq \tau+\varepsilon, t \geq t_{1}$. Evidently for $t \geq t_{1}$ the conditions of Theorem 1 hold: $\alpha \tau_{0}<\tau-\varepsilon$ and $(\tau+\varepsilon)\|b\|_{\left[t_{0}, \infty\right)}+\frac{\sigma\|a\|_{\left[t_{0}, \infty\right)}\|b\|_{\left[t_{0}, \infty\right)}\left(1-a_{0}\right)}{\left(1-\|a\|_{\left[t_{0}, \infty\right)}\right)^{2}}<\left(1-\|a\|_{\left[t_{0}, \infty\right)}\right)\left(1+\frac{\alpha}{e}\right)$.

Consider now two partial cases of equation (1.1), one with constant coefficients

$$
(x(t)-a x(g(t)))^{\prime}=-b(t) x(h(t)),
$$

where $a$ is a positive constant, and another with a non-delayed term

$$
(x(t)-a(t) x(g(t)))^{\prime}=-b(t) x(t)
$$

and assume that the suitable parts of conditions (a1), (a2), (a4) hold for these equations.

Corollary 3. Assume that for some $t_{0} \geq 0$ and $\alpha \in[0,1]$, for $t \geq t_{0}$ we have $\alpha \leq \frac{\delta e\|b\|_{\left[t_{0}, \infty\right)}}{1-a}$ and $\tau\|b\|_{\left[t_{0}, \infty\right)}+\frac{\sigma a\|b\|_{\left[t_{0}, \infty\right)}}{1-a}<(1-a)\left(1+\frac{\alpha}{e}\right)$. Then equation (2.9) is uniformly exponentially stable.

Corollary 4. If $a(t) \geq a_{0}>0, \frac{\sigma\|a\|_{\left[t_{0}, \infty\right)}\|b\|_{\left[t_{0}, \infty\right)}\left(1-a_{0}\right)}{\left(1-\|a\|_{\left[t_{0}, \infty\right)}\right)^{3}}<1$ then equation (2.10) is uniformly exponentially stable.

In Theorem 1 and its corollaries we assume that $a(t) \geq 0$. In the next theorem we remove this restriction. For any $u \in \mathbb{R}$ denote $u^{+}=\max \{u, 0\}, u^{-}=\max \{-a, 0\}$, hence $u=u^{+}-u^{-}$.

Define

$$
\bar{\tau}=\frac{1-\left\|a^{+}\right\|_{\left[t_{0},+\infty\right)}}{e\|b\|_{\left[t_{0},+\infty\right)}},\left(S^{+} y\right)(t)= \begin{cases}a^{+}(t) y(g(t)), & g(t) \geq t_{0}, \\ 0, & g(t)<t_{0} .\end{cases}
$$

Theorem 2. Assume that (a1),(a2),(a4) hold and there are $t_{0} \geq 0$ and $\alpha \in[0,1]$ such that for $t \geq t_{0}$, $\alpha \bar{\tau} \leq \delta$ and

$$
\tau\|b\|_{\left[t_{0}, \infty\right)}+\frac{\sigma\left\|a^{+}\right\|_{\left[t_{0}, \infty\right)}\|b\|_{\left[t_{0}, \infty\right)}}{\left(1-\left\|a^{+}\right\|_{\left[t_{0}, \infty\right)}\right)^{2}}+\frac{\left\|a^{-}\right\|_{\left[t_{0}, \infty\right)}\|b\|_{\left[t_{0}, \infty\right)}}{1-\left\|a^{+}\right\|_{\left[t_{0}, \infty\right)}}<1-\|a\|_{\left[t_{0}, \infty\right)}+\frac{\alpha\left(1-\left\|a^{+}\right\|_{\left[t_{0}, \infty\right)}\right)}{e} .
$$

Then equation (1.1) is uniformly exponentially stable.

Proof. We follow the proof of Theorem 1. Consider initial value problem (1.5) with $\|f\|_{\left[t_{0},+\infty\right)}<+\infty$, $f(t)=0$ for $t \in\left[t_{0}, t_{0}+\tau\right]$ and let $I=\left[t_{0}, t_{1}\right]$ for some $t_{1}>t_{0}+\tau$. By (1.7) the solution $x$ of (1.5) 
and the derivative of the function $y(t)=x(t)-a(t) x(g(t))=x(t)-a^{+}(t) x(g(t))+a^{-}(t) x(g(t))$ satisfy $y^{\prime}(t)=-b(t) x(h(t))+f(t), x=(E-S)^{-1} y=\left(E-S^{+}\right)^{-1} y-\left(E-S^{+}\right)^{-1}\left(a^{-}(\cdot) x(g(\cdot))\right)$ and

$$
|x|_{I} \leq \frac{1}{1-\|a\|_{\left[t_{0},+\infty\right)}}|y|_{I},\left|y^{\prime}\right|_{I} \leq \frac{\|b\|_{\left[t_{0},+\infty\right)}}{1-\|a\|_{\left[t_{0},+\infty\right)}}|y|_{I}+\|f\|_{\left[t_{0},+\infty\right)} .
$$

By Lemma 5. (1.5) is equivalent to the equation with an infinite number of delays

$$
y^{\prime}(t)=-b(t) \sum_{j=0}^{\infty} \prod_{k=0}^{j-1} a^{+}\left(h\left(g^{[k]}(t)\right)\right) y\left(h\left(g^{[j]}(t)\right)\right)+b(t)\left(E-S^{+}\right)^{-1}\left(a^{-}(t) x(g(t))\right)+f(t) .
$$

Denote

$$
\bar{B}(t)=b(t) \sum_{j=0}^{\infty} \prod_{k=0}^{j-1} a^{+}\left(h\left(g^{[k]}(t)\right)\right)
$$

where we can assume that $a(t)=0$ for $t<t_{0}$. Consider the delay equation

$$
x^{\prime}(t)+\bar{B}(t) x(t-\alpha \bar{\tau})=0 .
$$

where $\bar{\tau}$ is defined in (2.11).

Using the bounds for $a$ and $b$, we obtain $b_{0} \leq \bar{B}(t) \leq \frac{\|b\|_{\left[t_{0}, \infty\right)}}{1-\left\|a^{+}\right\|_{\left[t_{0}, \infty\right)}}$. Equation (2.13) can be rewritten in the form

$$
y^{\prime}(t)+\bar{B}(t) y\left(t-\alpha \tau_{0}\right)=b(t) \sum_{j=0}^{\infty} \prod_{k=0}^{j-1} a^{+}\left(h\left(g^{[k]}(t)\right)\right) \int_{h\left(g^{[j]}(t)\right)}^{t-\alpha \tau_{0}} y^{\prime}(\xi) d \xi+b(t)\left(E-S^{+}\right)^{-1}\left(a^{-}(t) x(g(t))\right)+f(t) .
$$

Since $\bar{B}(t) \geq b_{0}$ and $\alpha \bar{\tau} \bar{B}(t) \leq \frac{1}{e}$, by Lemma 4 equation (2.15) is exponentially stable, and its fundamental function is positive: $X_{1}(t, s)>0, t \geq s \geq t_{0}$.

By the same calculations as in Theorem 1 we have

$$
|y|_{I} \leq\left[\left(\tau-\alpha \bar{\tau}+\frac{\sigma\left\|a^{+}\right\|_{\left[t_{0}, \infty\right)}}{\left(1-\left\|a^{+}\right\|_{\left[t_{0}, \infty\right)}\right)^{2}}\right) \frac{\|b\|_{\left[t_{0}, \infty\right)}}{1-\|a\|_{\left[t_{0}, \infty\right)}}+\frac{\|b\|_{\left[t_{0}, \infty\right)}\left\|a^{-}\right\|_{\left[t_{0}, \infty\right)}}{1-\left\|a^{+}\right\|_{\left[t_{0}, \infty\right)}} \frac{1}{1-\|a\|_{\left[t_{0}, \infty\right)}}\right]|y|_{I}+M_{2},
$$

where the constant $M_{2}$ does not dependent on $I$.

The conditions of the theorem imply

$$
\left(\tau-\alpha \bar{\tau}+\frac{\sigma\left\|a^{+}\right\|_{\left[t_{0}, \infty\right)}}{\left(1-\left\|a^{+}\right\|_{\left[t_{0}, \infty\right)}\right)^{2}}\right) \frac{\|b\|_{\left[t_{0}, \infty\right)}}{1-\|a\|_{\left[t_{0}, \infty\right)}}+\frac{\|b\|_{\left[t_{0}, \infty\right)}\left\|a^{-}\right\|_{\left[t_{0}, \infty\right)}}{1-\left\|a^{+}\right\|_{\left[t_{0}, \infty\right)}} \frac{1}{1-\|a\|_{\left[t_{0}, \infty\right)}}<1 .
$$

Hence $|y(t)| \leq M$ for $t \geq t_{0}$, for some constant $M$ which does not depend on the interval $I$. Therefore $x$ is a bounded function on $\left[t_{0}, \infty\right)$. By Lemma 2 , equation (1.1) is uniformly exponentially stable.

Remark 2. If, in addition to the assumptions of Theorem Q $\|a\|_{\left[t_{0}, \infty\right)}=\left\|a^{+}\right\|_{\left[t_{0}, \infty\right)}$, which is equivalent to $\sup _{t \geq t_{0}} a(t) \geq \sup _{t \geq t_{0}}(-a(t))$, and

$$
\tau\|b\|_{\left[t_{0}, \infty\right)}+\frac{\sigma\|a\|_{\left[t_{0}, \infty\right)}\|b\|_{\left[t_{0}, \infty\right)}}{\left(1-\|a\|_{\left[t_{0}, \infty\right)}\right)^{2}}+\frac{\left\|a^{-}\right\|_{\left[t_{0}, \infty\right)}\|b\|_{\left[t_{0}, \infty\right)}}{1-\|a\|_{\left[t_{0}, \infty\right)}}<\left(1-\|a\|_{\left[t_{0}, \infty\right)}\right)\left(1+\frac{\alpha}{e}\right)
$$

then equation (1.1) is uniformly exponentially stable.

Assuming $\alpha=1$ and $\alpha=0$ in Theorem 2, we get the following tests. 
Corollary 5. Assume that conditions (a1),(a2),(a4) are satisfied, and for $t \geq t_{0}$ at least one of the following conditions holds:

a) $\bar{\tau}<\delta$ and

$\tau\|b\|_{\left[t_{0}, \infty\right)}+\frac{\sigma\left\|a^{+}\right\|_{\left[t_{0}, \infty\right)}\|b\|_{\left[t_{0}, \infty\right)}}{\left(1-\left\|a^{+}\right\|_{\left[t_{0}, \infty\right)}\right)^{2}}+\frac{\left\|a^{-}\right\|_{\left[t_{0}, \infty\right)}\|b\|_{\left[t_{0}, \infty\right)}}{1-\left\|a^{+}\right\|_{\left[t_{0}, \infty\right)}}<1-\|a\|_{\left[t_{0}, \infty\right)}+\frac{1-\left\|a^{+}\right\|_{\left[t_{0}, \infty\right)}}{e}$.

b) $\tau\|b\|_{\left[t_{0}, \infty\right)}+\frac{\sigma\left\|a^{+}\right\|_{\left[t_{0}, \infty\right)}\|b\|_{\left[t_{0}, \infty\right)}}{\left(1-\left\|a^{+}\right\|_{\left[t_{0}, \infty\right)}\right)^{2}}+\frac{\left\|a^{-}\right\|_{\left[t_{0}, \infty\right)}\|b\|_{\left[t_{0}, \infty\right)}}{1-\left\|a^{+}\right\|_{\left[t_{0}, \infty\right)}}<1-\|a\|_{\left[t_{0}, \infty\right)}$.

Then equation (1.1) is uniformly exponentially stable.

In the following theorem, we do not assume boundedness of delays.

Theorem 3. Assume that (a1)-(a3) hold, $\int_{t_{0}}^{\infty} b(s) d s=\infty, b(t) \neq 0$ almost everywhere, and there exist $t_{0} \geq 0, \alpha>0, \tilde{\delta} \geq 0, \tilde{\tau}>0$ and $\tilde{\sigma}>0$ such that for $t \geq t_{0}$,

$$
\tilde{\delta} \leq \int_{h(t)}^{t} b(\xi) d \xi \leq \tilde{\tau}, \int_{g(t)}^{t} b(\xi) d \xi \leq \tilde{\sigma}, \alpha \tilde{\tau}_{0} \leq \tilde{\delta}, \tilde{\tau}_{0}:=\frac{1-\|a\|_{\left[t_{0}, \infty\right)}}{e}
$$

and

$$
\tilde{\tau}+\frac{\tilde{\sigma}\|a\|_{\left[t_{0}, \infty\right)}\left(1-a_{0}\right)}{\left(1-\|a\|_{\left[t_{0}, \infty\right)}\right)^{2}}<\left(1-\|a\|_{\left[t_{0}, \infty\right)}\right)\left(1+\frac{\alpha}{e}\right) .
$$

Then equation (1.1) is asymptotically stable.

Proof. We follow the scheme of the proof for Theorem 1, Denote $y(t)=x(t)-a(t) x(g(t))$. Then (1.1) is equivalent to the equation with an infinite number of delays:

$$
y^{\prime}(t)=-b(t) \sum_{j=0}^{\infty} \prod_{k=0}^{j-1} a\left(h\left(g^{[k]}(t)\right)\right) y\left(h\left(g^{[j]}(t)\right)\right),
$$

where

$$
g^{[0]}(t)=t, g^{[1]}(t)=g(t), g^{[k]}(t)=g\left(g^{[k-1]}(t)\right), k \in \mathbb{N}
$$

Let $s=p(t):=\int_{t_{0}}^{t} b(\tau) d \tau, s \geq 0, z(s)=y(t)$, where $p(t)$ is a strictly increasing function. Then we introduce $\tilde{h}(s)$ and $\tilde{g}(s)$ as follows:

$$
\begin{gathered}
y(h(t))=z(\tilde{h}(s)), \tilde{h}(s) \leq s, \tilde{h}(s)=\int_{t_{0}}^{h(t)} b(\tau) d \tau, s-\tilde{h}(s)=\int_{h(t)}^{t} b(\tau) d \tau, \\
\tilde{g}(s)=\int_{t_{0}}^{g(t)} b(\tau) d \tau, s-\tilde{g}(s)=\int_{g(t)}^{t} b(\tau) d \tau, \tilde{g}(s) \leq s \\
\dot{y}(t)=b(t) \dot{z}(s), \dot{y}(g(t))=b(g(t)) \dot{z}(\tilde{g}(s)) .
\end{gathered}
$$

Equation (2.18) can be rewritten in the form

$$
z^{\prime}(s)=-\sum_{j=0}^{\infty} \prod_{k=0}^{j-1} a\left(\tilde{h}\left(\tilde{g}^{[k]}(s)\right)\right) z\left(\tilde{h}\left(\tilde{g}^{[j]}(s)\right)\right) .
$$

Consider the initial value problem

$$
z^{\prime}(s)=-\sum_{j=0}^{\infty} \prod_{k=0}^{j-1} a\left(\tilde{h}\left(\tilde{g}^{[k]}(s)\right)\right) z\left(\tilde{h}\left(\tilde{g}^{[j]}(s)\right)\right)+f(s), z(s)=0, s \leq 0
$$


with $\|f\|_{[0,+\infty)}<+\infty, f(s)=0$ for $s \in[0, \tilde{\tau}]$.

Problem (2.20) has a form of problem (2.4) with $b(t) \equiv 1$, where condition (2.17) corresponds to (2.2). As in the proof of Theorem 1, one can show that the solution $z$ of (2.20) is a bounded on $\left[t_{0}, \infty\right)$ function. Then equation (2.19) is exponentially stable. Hence for any solution $z$ of (2.19), $\lim _{s \rightarrow \infty} z(s)=0$. If $y$ is a solution of (2.18) then $\lim _{t \rightarrow \infty} y(t)=\lim _{s \rightarrow \infty} z(s)=0$. If $x$ is a solution of (2.4) then $x=(E-S)^{-1} y$, therefore $\lim _{t \rightarrow \infty} x(t)=0$ as well. Finally, equation (1.1) is asymptotically stable.

3. Examples and Conclusion. First, we compare the results of the present paper with Propositions 1 and 2 .

Example 1. Consider an equation with constant delays and variable coefficients

$$
(x(t)-(0.498+0.001 \cos t) x(t-\pi))^{\prime}=-r(0.9+0.1 \sin t) x(t-\pi),
$$

which allows comparison to known results. Denote $\bar{r}:=\limsup _{t \rightarrow \infty} r \int_{t-\pi}^{t}(0.9+0.1 \sin s) d s=r(0.9 \pi+2)$. Here $a_{0}=0.497, A_{0}=0.499$. Corollary 1 b) implies exponential stability for $0.059<\bar{r}<0.109$, while part a) gives $0<\bar{r}<0.0797$, which overall gives exponential stability for $\bar{r}<0.109$. Since $\sqrt{2\left(1-2 A_{0}\right)} \approx 0.0632$,

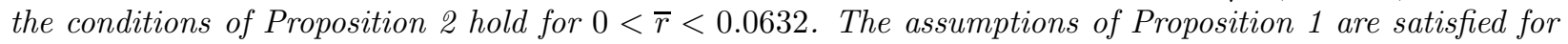
$0<\bar{r}<0.002$, so Corollary 1 of Theorem 1 leads to a sharper result.

Next, we illustrate the results of the paper with examples for which all previous tests fail. In the following two examples, we outline the role of $\alpha$ in Theorem 1 .

Example 2. Consider the equation

$$
(x(t)-0.6 x(t-0.2|\sin t|))^{\prime}=-x(t-0.14) .
$$

Here $a(t)=0.6,\|b\|_{\left[t_{0}, \infty\right)}=1, \tau=\delta=0.14, \sigma=0.2$. Condition (2.8) in Corollary 0 for equation (3.2) has the form

$$
\frac{0.4 \alpha}{e} \leq 0.14<0.1+\frac{0.4 \alpha}{e} .
$$

For $\alpha \in(0.1 e, 0.35 e] \approx(0.272,0.951]$ condition $(3.3)$ holds, in particular for $\alpha=0.5$. Hence by Corollary 2 equation (3.2) is uniformly exponentially stable. Note that for $\alpha=0$ and for $\alpha=1$ condition (3.3) fails.

Propositions 1 and Q are not applicable, as the delay of the neutral term is variable. Moreover, the coefficient $a(t)$ in the neutral part in Propositions 1 and 2 must be less than 0.5, while in (3.3) $a(t)=0.6>0.5$.

Example 3. Consider an equation with variable coefficients and delays

$$
(x(t)-(0.5+0.1 \cos t) x(g(t)))^{\prime}=-r(0.9+0.1 \sin t) x(t-1), 0.9 \leq t-g(t) \leq 1 .
$$

We have $\tau=\sigma=\delta=1, a_{0}=0.4,\|a\|_{\left[t_{0}, \infty\right)}=0.6,\|b\|_{\left[t_{0}, \infty\right)}=r$. Then the assumptions of Theorem 1 are equivalent to

$$
\alpha \frac{0.4}{e r} \leq 1, r+r \frac{0.6^{2}}{0.4^{2}}<0.4\left(1+\frac{\alpha}{e}\right) \Leftrightarrow \frac{0.4 \alpha}{e} \leq r \leq 0.4 \frac{4}{13}\left(1+\frac{\alpha}{e}\right) .
$$

This corresponds to the values between two lines in $(\alpha, r)$ graph on Fig. 1. The highest allowed value of $r$ is achieved for $\alpha=1$ and corresponds to Part a) of Corollary 1, $r<r_{0} \approx 0.168$. To the best of our knowledge, other known tests are not applicable to (3.4).

Example 4. Consider an equation with an oscillating coefficient in the neutral term

$$
(x(t)-0.6(\sin t) x(t-0.2|\cos t|))^{\prime}=-0.1(0.5+|\sin t|) x(t-0.5) .
$$




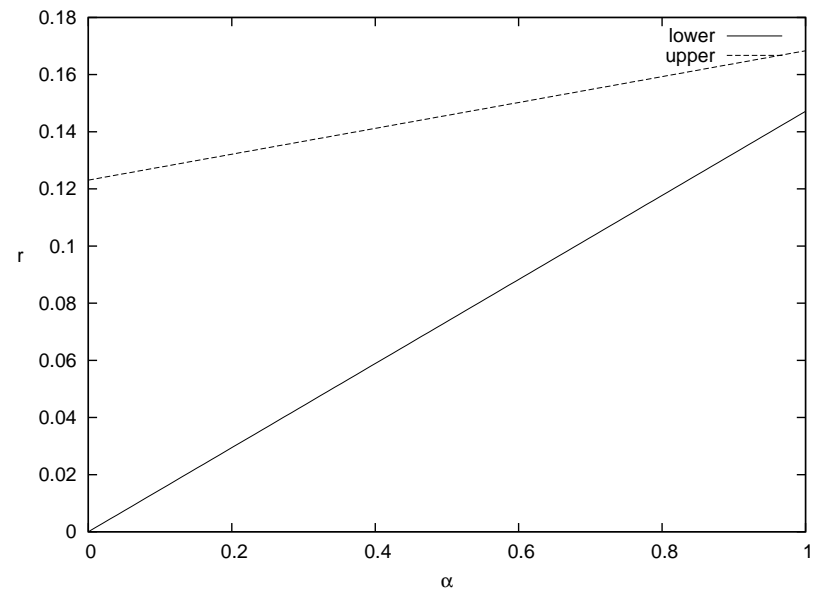

Figure 1: The lower and the upper bounds for $r$ deduced from Theorem 1 for which equation (3.4) is asymptotically stable for any $\alpha \in[0,1]$.

Here

$$
\|a\|_{\left[t_{0}, \infty\right)}=\left\|a^{+}\right\|_{\left[t_{0}, \infty\right)}=\left\|a^{-}\right\|_{\left[t_{0}, \infty\right)}=0.6,\|b\|_{\left[t_{0}, \infty\right)}=0.15, \tau=0.5, \sigma=0.2 .
$$

The inequality in Theorem 2 has the form $0.4125<0.4+0.147 \alpha$ which holds for $\alpha>0.085$. Note that, as $\delta=\tau=0.5$ and $\bar{\tau} \approx 0.98$ in (2.11), any $\alpha<0.5$ satisfies $\alpha \bar{\tau}<\delta$. For $\alpha=0.45$ we get the correct inequality $0.4125<0.4147$, hence Equation (3.5) is uniformly exponentially stable.

Propositions 1, 圆 and Theorem 1 fail for Equation (3.5).

Finally, we apply Theorem 3 to an equation with unbounded delays.

Example 5. Consider a pantograph-type neutral equation

$$
\left(x(t)-0.55 x\left(\frac{t}{3}\right)\right)^{\prime}=-\frac{1}{4 t} x\left(\frac{t}{2}\right), t \geq t_{0}>0 .
$$

Then in Theorem 3 we have $\tilde{\sigma}=0.25 \ln 3, \tilde{\delta}=\tilde{\tau}=0.25 \ln 2, \tau_{0} \approx 0.1655<\tilde{\delta} \approx 0.173$, so any $\alpha$ can be used in (2.16). Any $\alpha \geq 0.36$ implies asymptotic stability; in particular, for $\alpha=1$ inequality (2.17) has the form $0.509<0.6$ and thus holds.

In the present paper, we considered neutral equation (1.1) in the most general framework: all coefficients and delays are measurable functions. We also do not assume coefficient in the neutral part $\|a(t)\|<\frac{1}{2}$, which is usually imposed, see for example Propositions 1 and 2. In four of the five examples, $a(t)$ can exceed 0.5.

The method used in the paper is based on the Bohl-Perron theorem and a transformation of the given neutral equation to an equation with an infinite number of delays. This scheme is applied to neutral equations in the Hale form for the first time, and we illustrated its efficiency.

Acknowledgment. The second author was partially supported by the NSERC research grant RGPIN2015-05976.

\section{REFERENCES}

1. N. V. Azbelev, L. M. Berezanski and L.F. Rahmatullina, A linear functional-differential equation of evolution type (Russian), Differencialnye Uravnenija 13 (1977), 1915-1925, 2106.

2. N. V. Azbelev and P. M. Simonov, Stability of Differential Equations with Aftereffect. Stability and Control: Theory, Methods and Applications, 20. Taylor \& Francis, London, 2003. 
3. L. Berezansky and E. Braverman, Explicit stability conditions for linear differential equations with several delays, J. Math. Anal. Appl. 332 (2007), 246-264.

4. E. Fridman, Introduction to time-delay systems. Analysis and control. Systems \& Control: Foundations \& Applications. Birkhuser/Springer, Cham, 2014.

5. M. I. Gil', Stability of Neutral Functional Differential Equations. Atlantis Studies in Differential Equations, 3. Atlantis Press, Paris, 2014.

6. K. Gopalsamy, Stability and Oscillations in Delay Differential Equations of Population Dynamics. Mathematics and its Applications, 74. Kluwer, 1992.

7. I. Györi and G. Ladas, Oscillation Theory of Delay Differential Equations. Clarendon Press, Oxford, 1991.

8. J. Hale, Theory of Functional Differential Equations (second edition), Applied Mathematical Sciences, Vol. 3. SpringerVerlag, New York-Heidelberg, 1977.

9. V. B. Kolmanovskii and A. D. Myshkis, Introduction to the Theory and Applications of Functional-Differential Equations. Mathematics and its Applications, 463. Kluwer, Dordrecht, 1999.

10. Y. Kuang, Delay Differential Equations with applications in Population Dynamics, Academic Press, Boston, San Diego, 1993.

11. X. H. Tang and X. Zou, Asymptotic stability of a neutral differential equations, Proc. Edinb. Math. Soc. (2) 45 (2002), 333-347.

12. J.S. Yu, Asymptotic stability for non-autonomous scalar neutral differential equations, J. Math. Anal. Appl. 203 (1996), $850-860$.

Department of Mathematics, Ben-Gurion University of the Negev, Beer-Sheva 84105, Israel

Email address: brznsky@math.bgu.ac.il

Department of Mathematics and Statistics, University of Calgary, 2500 University Drive N.W., Calgary, AB T2N

1N4, CANADA

Email address: maelena@ucalgary.ca 